\title{
Certainty in ascending sensory signals- the unexplored driver of analgesic placebo response
}

\author{
P. Kuperman $\mathrm{PhD}^{1}$, D. Talmi $\mathrm{PhD}^{2}$, NP Katz $\mathrm{MD}^{3}$, R. Treister $\mathrm{PhD}^{1,4^{*}}$
}

1. The Clinical Pain Innovation Lab, University of Haifa, Haifa, Israel

2. Department of Psychology, University of Cambridge

3. WCG Analgesic Solutions, Wayland, MA, USA; Department of Anaesthesiology and Perioperative Medicine, Tufts University School of Medicine, Boston, Massachusetts, United States of America.

4. Faculty of Social Welfare and Health Sciences, University of Haifa, Israel

* Corresponding author.

Address: University of Haifa, 199 Aba Khoushy Ave, Mount Carmel, Haifa, Israel, Tel.: 972-533-839935; Fax: 972-4-9529926.

E-mail address: treister.roi@gmail.com. 


\begin{abstract}
Previous frameworks have failed to adequately explain the observed correlation between withinsubject variability in pain reporting and analgesic placebo response. These relationships have been observed in both clinical and experimental setups.

Within-subject variability of clinical pain scores is traditionally assessed based on daily pain diaries collected during the pre-intervention stage. Experimental variability can be assessed by the Focused Analgesia Selection Test (FAST), which calculates the relationship between noxious stimuli administrated at various intensities and pain reports. The variability, either clinical or experimental, has been shown to predict the placebo response.

In explaining the placebo response, Bayesian Brain Hypothesis $(\mathrm{BBH})$ posits that pain perception (posterior), is composed of certainty (precision) of expectations (priors due to belief or conditioning) and incoming sensory information (likelihood), with the bulk of research focused on the precision of priors. Virtually all placebo analgesia research has focused on the priors and their certainty, rather than on the certainty of the likelihood, mainly because it cannot be assessed directly.

We propose that the within-subject variability, as encapsulated by the FAST, is a proxy for certainty in (or, precision of) ascending sensory signals, and our results suggest that it could not only be assessed, but also manipulated. If true, our hypothesis will facilitate new lines of research and could potentially promote precision analgesic medicine by use of variability of pain scores as a diagnostic method to identify pain patients who will benefit from specific treatments.
\end{abstract}




\section{BACKGROUND}

Overview

In this article, we offer a comprehensive understanding of the relations between within-subject variability of pain reports, the analgesic placebo response and the Bayesian Brain hypothesis $(\mathrm{BBH})$. Specifically, we argue that measures of variability could represent a less explored facet of the BBH. By tying concepts and definitions from these domains together, we offer a more comprehensive model explaining changes in the perception of pain, and discuss its clinical relevance.

\section{Introduction to the placebo}

A placebo is an inert treatment with no specific therapeutic properties, wherein the placebo effect is the term applied to the response to the inert treatment. The placebo response is the measurable improvement in symptoms which come about due to the administration of a placebo [1]. Given that much of placebo research has centered around the analgesic placebo response, the current manuscript will focus on placebo analgesia in the clinical and experimental settings. Decades of study on placebo have shown that a variety of placebo 'responses' can ensue from its introduction, including reported changes to subjective symptoms (e.g. pain, mood) [2,3], which have been correlated with underlying neurobiological underpinnings [4,5] and potential genetic markers [6,7].

The prevailing consensus is that the placebo 'response' is resultant both from the placebo treatment itself and the context in which it was delivered. The magnitude of the response is further mediated by expectations, which are derived from previous experience (i.e. 
conditioning), instructions, and social learning [8,9] as well as behaviors embedded in the patient-physician relationship, such as warmth, attention, tone of voice and bodily expressiveness. [10]. According to the additivity theory, which is the currently-accepted view upon which the concept of Randomized Controlled Trials (RCTs) was developed, the response to a drug is the sum of the non-specific (read: placebo) aspects of the treatment and the specific pharmacologic aspects with the proviso that the non-specific aspect is equal to the response that would be seen if only a placebo had been administered. This places the placebo response as an integral, non-negligible component of the response to any treatment, either active or placebo [10].

In the early stages of placebo research, the focus was mainly on the properties of the placebo manipulation itself - the shape, size, color $[12,13,14]$ and route of administration $[15,16]$ have all been shown to modify the placebo effect. Since then, the emphasis has shifted to the characteristics of the patient (e.g. expectations) [17], the healthcare provider and their interaction with the patient (the "healing ritual") $[10,18]$ and even the interaction between patients themselves [19].

Given the myriad of insights and knowledge which were accumulated over the past three decades, experts are now looking to harness the placebo effect to improve outcomes: where in clinical care, the aim is to try to increase its effects for the benefits of patients [20], and in clinical drug development, the aim is to reduce the effect, to support improved assay sensitivity, namely the ability of a trial to demonstrate the benefit of truly efficacious treatment $[11,21]$. If the additivity theory is true, the latter aim could not be achieved, because successfully reducing the magnitude of placebo response in a trial would result in the same reduction in the 
effect of the active treatment, hence no change would be seen in the treatment effect (which is, the change in treatment arm minus the change in placebo arm). Indeed, the additivity theory has started to be challenged, for example by the interactive model [see 11]. Where the additivity model assumes equal sized effects of the placebo in both the placebo and the drug groups, the interactive model assumes that drug-specific effects may interact with the placebo responses, leading to unequal placebo effects in the two groups [11]. Furthermore, it should be noted that excluding placebo responders will not necessarily improve assay sensitivity - to achieve that one should exclude those who show a good response to placebo but not to the drug -a subgroup that could best be described as "preferential placebo responders."

While it was initially assumed that only a portion of the population was placebo-sensitive, the emergent research has suggested that almost all individuals can be, in one situation or another, placebo responders [22]. Hence, the current working model places greater emphasis on the situational or fluctuating determinants of the placebo response [23]. Situational determinants can be generated both at the level of the environment (e.g. manipulation of contextual cues) or at the level of the individual (e.g. manipulation of mood) [3].

Within-subject variability of clinical pain reports as a predictor of the placebo response

While a few predictors, such as sex or optimism, have sporadically been found to predict the placebo response, none have been consistent [23]. The most often-cited factor to predict the analgesic placebo response is the within-subject variability of daily clinical pain reports, which represents the individual fluctuations in day-to-day pain of chronic pain patients. 
The first to demonstrate the idea that vacillations in reported pain predict the placebo response was Harris et al [24] in a retrospective analysis of data from fibromyalgia patients. They calculated the within-subject fluctuations in terms of individual standard deviation of daily pain scores, as obtained from pain diaries completed at baseline, prior to the initiation of study treatment. They found that the individual standard deviation (termed "pain variability index") predicted patient response to placebo, but not to the drug. This finding was later confirmed in a meta-analysis of 12 clinical trials in postherpetic neuralgia and painful diabetic peripheral neuropathy [25]. Since the publication of this meta-analysis, it has been often recommended to exclude patients who exhibit large day-to-day variability from analgesic clinical trials [26] as they have an increased likelihood of responding to placebo, but not to active treatment, thus compromising the assay sensitivity of the trial [27]. It seems that the relationship between within-subject fluctuations and the placebo response might not be specific to pain. For example, in a retrospective analysis, Zilcha-Mano and Barber [28] reported that the larger the change between the two time points of depression evaluation which took place during the screening period (prior to any treatment), the larger the placebo response.

Although expectations for benefit from treatment and conditioning due to past experiences are regarded as the main drivers of the placebo response, they are seemingly unrelated to the variability of pain scores. Hence, despite the importance of all three abovementioned factors, current placebo theory does not explain the relationship between them.

Within-subject variability of experimental pain reports as a predictor of the placebo response 
In an attempt to learn more about people's tendency to demonstrate fluctuations in perceived pain, we developed a laboratory analogue to pain diaries, a method termed the Focused Analgesia Selection Test (FAST), which assesses the within-subject variability in response to experimentally-induced noxious stimuli of various stimuli intensities [29]. More specifically, the FAST procedure includes exposure to noxious stimuli, administrated repeatedly at several different intensities, in a randomized and blinded design. Small adjustments are made to the location of the applied stimuli to minimize sensitization and/or habituation effects. Pain intensity ratings are obtained from the participant after each stimulus on a 0-100 numerical rating scale (NRS) ranging from 0 ("no pain") to 100 ("worst pain imaginable"). Relationship between stimulus intensities and pain reports are calculated ( $\mathrm{R}^{2}$ and ICC) to assess the magnitude of variability observed. When the reported pain intensities are concurrent with the intensity of the applied stimuli, low variability is observed (see example in Fig 1A).

The FAST was implemented in a series of studies. In the first [29], we asked a cohort of osteoarthritis (OA) patients to report their pain before and after an exercise in which the affected joint is moved. We know that on average, patients with OA of the knee will report an increase in pain following such an exercise. We hypothesized that the FAST results will positively correlate with the increase in clinical pain - because it is reasonable to assume that subjects who show reduced variability in the FAST will better report the change in their clinical pain. The results of the experiment were in line with our hypothesis; this was the first hint that the FAST measures a construct which is clinically relevant. Next, we wanted to learn if variability of reported pain during the FAST procedure could be reduced through training. To achieve that, we conducted an RCT in painful diabetic neuropathy [30], in which half of the cohort underwent training aimed to reduce variability of pain reports. The training was based on preforming the FAST while 
receiving feedback on performance. After the training, all patients entered the treatment phase. As predicted, variability in the FAST was reduced in the trained arm. An unpredicted result was the effect of the training on the treatment response: while both trained and untrained patients responded to the drug similarly, the trained patients demonstrated significantly decreased placebo response, even though the training does not include aspects that might modulate expectation for pain (or pain relief). These results, in which the FAST predicted changes in spontaneous pain (compared to the evoked pain in the OA study) further extended the external validity of our approach. In a third study [31], we found that the FAST results were correlated with the variability of day-to-day clinical pain ratings, and both correlated with the placebo response where variability of experimental pain reports, as measured by FAST, predicted preferential placebo responsiveness. Implementing the FAST either as a screening tool aimed to identify and exclude candidates who are expected to show preferential placebo response, or implementing as a training aimed to reduce variability of pain scores in drug development programs could theoretically improve the assay sensitivity of analgesic trials. Regarding the duration of the effects of the training, evidence supports that effects last for at least several $(\sim 4)$ weeks [30], while longer effects were not yet tested. It is possible that in the context of chronic pain trials, in which the treatment period is commonly 12 weeks or more, training should be repeated to maintain its effects throughout the study.

To summarize, mounting evidence suggests that large variability in experimental pain, as measured by the FAST, is associated with large variability of day-to-day clinical pain, and both can predict the placebo response. (see Figure 2).

Bayesian brain and prediction processing model $(B B H)$ 
The Bayesian brain and prediction processing model (also termed the Bayesian Brain Hypothesis, $\mathrm{BBH})$ is an emerging theory at the intersection of cognitive science and computational biology [32,33], which attempts to explain the effects of expectation on perception of various sensory modalities $[9,34,35,36]$. While once applied mainly to visual and auditory perception, the $\mathrm{BBH}$ has been suggested to be useful in understanding observed variability of other perceptual domains, including pain.

According the $\mathrm{BBH}$, which is based on probabilistic estimation, perception (posteriors) is not a direct reflection of reality. Rather, expectation, based on past and present experiences, including the representation of consciously-perceived and subliminal cues, previous associative learning, and stored beliefs (prior function) can bias perceptions away from the actual state of sensory information (likelihood function). The statistical confidence (will be used synonymously with precision) one has in those cues and beliefs (i.e. precision of the prior function) and in the state of sensory information (i.e. precision of the likelihood function) determine how much their perception will deviate from the sensory input; where precision is represented by the inverse of statistical variance of the function (Fig 3a). The larger the confidence in the priors, the more perception will shift toward expectations, (Fig 3b), and the larger the confidence in the likelihood, the less perception will shift toward expectations (Fig 3c). The gap between incoming information (the likelihood) and the expectation (prior) is termed the "prediction error". The eventual perception (posterior) reflects an updating of the priors, based on the recent likelihood. Later, it forms the prior of subsequent experiences, allowing the agent to predict the next event more accurately. Thus, BBH provides a mathematically precise way to formalize the broad understanding of perception as the result of the integration of bottom-up ascending signals from 
the periphery and top-down descending signals from brain regions that represent multi-modal associations.

Recent studies have demonstrated that the Bayesian model explains pain perception better than other models $[34,40,41]$. This is in line with studies which have been performed $[37,38]$ affirming the connection between precision of priors and pain expectations. Using $\mathrm{BBH}$, we can interpret the studies on the basis of the assumption that expectations for benefit from a placebo treatment, which are based on past experience and conditioning, shape the priors; that the likelihood function corresponds to the noisy representation of the actual ascending sensory signals; and that reported levels of pain following the placebo treatment provide a rough read-out of the posteriors. For example, Colloca et.al (2010) performed a study among healthy controls, aimed to assess the effect of repeated conditioning on the placebo response. They found that the more exposures a participant had to the conditioning paradigm, the longer the placebo lasted. In BBH terminology, Colloca et. al's intervention increased subjects' confidence in their priors, resulting in a larger shift of perception towards them. Likewise, using imaging techniques they [39] noted that a buildup of fMRI signal changes in the frontal cortex related to repeated conditioning, further supporting this connection.

In the aforementioned studies, the investigators are taking advantage of experimental pain paradigms, since they allow control over precise stimuli, which will in turn elicit an internal representation of the stimuli (likelihood). Those elicited representations could be more, or less, precise for the subject (precision of likelihood). Once the likelihood is known (controlled), assessing the effect of manipulations of expectations (priors) on the perceived pain (the posteriors) is straightforward. In the most recent study, Hoskin et al., 2019, using a cued pain 
task and Bayesian modeling, observed that the best model fit was one that varied the parameters based on each individual, suggesting that substantial inter-subjects differences exist, and can be useful in distinguishing individuals based on their sensitivity to pain expectations (priors).

Taken together these results suggest that $\mathrm{BBH}$ is an appropriate framework to model the placebo response, where higher confidence (precision) in expectations (i.e. priors constructed based on belief or conditioning) leads to an increased impact on pain perception.

\section{THE HYPOTHESIZED METHOD}

To date, the focus of analgesic placebo research has centered on the way in which priors and confidence in them shape the placebo response, while virtually no attention was given to the precision of the likelihood. This is mainly due to the fact that there are currently no established measures with which to estimate the precision of the likelihood.

Our hypothesis is that variability of pain scores, and particularly, the variability observed in the FAST procedure is a proxy measure for the precision of the ascending sensory signals (likelihood). Within this framework of the $\mathrm{BBH}$ - the connection between the performance in the FAST and the placebo response is intuitive: When expectations (priors) are kept constant, individuals who demonstrate lower variability of pain reports (better precision of likelihood), will show less shift away from the actual sensory information. Furthermore, upon training aimed to reduce variability in the FAST [30], which does not involve modulation of expectations, the placebo effect is diminished. 
This hypothesis, if true, will expand our ability to investigate factors which are contributing to the placebo response by permitting assessment and manipulation of the precision of the likelihood function.

\section{EVALUATION OF THE HYPOTHESIS}

Given that pain is a subjective measure with no objective gold-standards, and that ascending signals are only a component of the process that gives rise to the subjective experience of pain, it is impossible to assess our hypothesis directly. However, there are several investigational approaches that could indirectly support our claim.

Although far from perfect, there are well-known correlations between objective biomarkers, and subjective pain reports. Take for example the correlation between skin conductance and subjective pain reports [42], or gamma-band event-related synchronization ( $\gamma$-ERS) and withinsubject pain variability [43]. When a subject experiences pain, their sweat glands are activated, and fluctuations in skin conductance can be measured. Electrophysiological measures, such as $\gamma-$ ERS have been observed as well to selectively predict within-subject variability specifically for painful stimuli [43]. As such, it would be interesting to compare the correlations between the two among FAST performers with high vs. low variance. This could indirectly validate the FAST as a proxy measure for the precision of ascending sensory signals. With this, the FAST could be studied alongside measures of interoception, such as the heartbeat perception task, or multidimensional assessment of interoceptive awareness questionnaire (MAIA), in order to test if those who present low variability in the FAST will also demonstrate higher levels of interoceptive ability. Similarly, possible relations between the FAST and other psychological or 
cognitive constructs, such as awareness to bodily sensations (Body Awareness Questionnaire (BAQ) [44], the Self-Consciousness Scale-Revised (SCS-R) [45]), self-esteem (The Self-Esteem Stability Scale (SESS) [46]) and optimism (Life Orientation Test-Revised (LOT-R) [47]) should be investigated.

Second, in order to assess the potential relationship between FAST and Bayesian hierarchical modeling, it would be beneficial to perform both the FAST and a task which is based on the $\mathrm{BBH}$, such as the cued pain task [34] in future studies. This framework would serve a dual purpose, both to test the Bayesian model's ability to predict the outcome of the FAST, and to test the validity of the cued pain task against an already established instrument with clinical relevance. Third, in order to assess the FAST as a proxy measure for precision in ascending sensory signals it would be prudent to devise an experiment in which the certainty of the pain stimulus can be manipulated. By adding 'noise' or fluctuation, for example by applying several different temperatures within the same stimulus block, a participant's ability to judge incoming sensory information can be ascertained based on the pain intensity ratings that they provide. Fourth, while it has already been demonstrated in a small number of studies [30,31], it is worthwhile to incorporate the FAST technique into larger studies performed on diverse clinical populations. This setup would allow for the validation of previous findings connecting FAST, fluctuations in clinical pain and the placebo response.

\section{CONSEQUENCES OF THE HYPOTHESIS}

Our hypothesis opens new opportunities to investigate factors affecting the subjective experience of pain and with-it drivers of the placebo response.

In the past decade there has been an extensive body of evidence searching for biomarkers of pain and its modulation, and more specifically, of the placebo response. Studies from both healthy 
controls and chronic pain patients have highlighted brain areas in which activation has been found to be related to placebo analgesia. These works focus largely on the expectation of benefit, or pain anticipation [48]. Studying healthy controls, Roy et. al (2014) suggested that activation in the PAG is a biomarker for the prediction error of aversive stimuli [49]. Building on these findings, Grahl, Onat and Büchel (2018) noted that, in a Bayesian framework, the PAG represents precision of expectations (priors) and its signal change correlated with placebo effects on a behavioral level [50]. As the PAG is involved in both the ascending and descending pain system it could be hypothesized that subjects who are more tuned to their ascending sensory signals (i.e. those who exhibit low variability in the FAST) will also show reduced activation in the PAG in response to painful stimuli.

In addition to the PAG the most consistent placebo-related biomarkers include prefrontal areas such as dorsolateral, ventrolateral, and medial prefrontal cortex (dlPFC/vlPFC/mPFC), medial orbitofrontal cortex (OFC), mid-lateral OFC, and right midfrontal gyrus (r-MFG) [51,52,53,54]. For example Hashmi et.al (2012) observed that among chronic back pain patients two prefrontal functional connections synergistically predicted placebo analgesia; where one, left dorsal lateral prefrontal cortex-left midcingulate cortex(LdlPFC-LmCC) identified placebo responders, and the other, right dorsomedial prefrontal cortex- left midcingulate cortex (RdmPFC-LaINS) non-responders [54]. Alongside the neural markers identified by this research, psychological components, such as interoceptive awareness were also found to predict placebo response [52], reinforcing their utility in future FAST work.

While Lui et al.s (2010) work suggested that activation in the PAG could be regarded as a proxy measure for the precision of the priors (in Bayesian terminology); given that our hypothesis is that the FAST is a proxy for the precision of the likelihood, we do not necessarily anticipate 
correlations between FAST results and activation in the aforementioned areas. Rather, we hypothesize that these two measures of precision will explain different aspects of the variability of the placebo response. For example, a study which can assess priors, likelihoods, and their precision, using a computational model, can be used to more directly link biomarkers to these quantities. Research [55,56] summarizing the search for pain biomarkers in the brain notes that future studies should focus on assessing biomarker performance on an individual level, and in conjunction with tools based on pain-related behaviors that are easier to implement in a clinical setting. This future directive lends itself to non-invasive measures like the FAST. To conclude, we posit that the results of the FAST procedure could complement and improve the prediction of other biomarkers of placebo analgesia.

The main clinical relevance of our hypothesis is the potential to use variability of pain scores as a diagnostic method to identify pain patients who will benefit from specific treatment (i.e. personalized pain medicine). For example, take two chronic pain patients, suffering from the same pathology and expressing the same pain intensity (70/100). In the case of one, the expectations (priors) are for pain of 80/100 (e.g. I am afraid that it's going to hurt), the actual ascending signal is a 40/100, but because the certainty of the likelihood is low, pain is perceived at a level closer to that of the expectations (70/100) (Fig 4A). For such a patient, training that is focused on direction of attention towards the body (e.g. mindfulness) should theoretically increase certainty in the likelihood and consequently reduce their perceived pain level. In contrast, the other patient expects lower pain (40/100), but with low certainty of priors and an ascending signal of 80/100 hence their experience is closer to that of the likelihood (70/100) (Fig 4B). Such a patient will perhaps better benefit from behavioral therapy, such as cognitive behavioral therapy (CBT), which focuses on boosting expectations for benefit from treatment. 
Our hypothesis is aligned with Bergh et.al (2017) who suggest that "medically unexplained symptoms" (MUS) might be, at least in some patients, due to low certainty of the likelihood. Hence, interventions aimed to improve it, will also improve the symptom.

In conclusion, pain perception and placebo analgesia has been extensively studied. One model that was recently adopted to explain both is the Bayesian Brain Hypothesis. Still, there are no explanations for the robust correlation seen between an individual's tendency to demonstrate day-to-day fluctuations in pain and magnitude of placebo analgesia.

The fact that variability of pain scores in response to experimental pain correlated with day-today fluctuations in pain and both predict the placebo response implies that variability of pain (regardless of its origin, experimentally induced, or of clinical pain) might be a proxy measure for one's certainty (or confidence) in ascending sensory signals (i.e. likelihood). If true, our hypothesis will facilitate new studies and could potentially promote personalized pain management for clinical populations. Further research is warranted to assess the generalizability of our findings to other, non-painful conditions which depends on the subjective evaluation of symptoms. 


\section{ACKNOWLEDGMENTS}

We wish to thank Dr. Rita Canaipa for her insightful comments on the manuscript and Mr. El-Ad Cohen for his contribution to the figure designs.

\section{FUNDING}

The manuscript was funded by internal investigator funding. No external funding was provided. Therefore, no outside involvement occurred in the collection, analysis and interpretation of data; in the writing of the manuscript; or in the decision to submit the manuscript for publication.

\section{CONFLICT OF INTEREST STATEMENT}

All authors have no conflicts of interest to disclose. 


\section{REFERENCES}

1. Benedetti F. Placebo effects: from the neurobiological paradigm to translational implications. Neuron. 2014;84(3):623-37.

2. Colloca L. The placebo effect in pain therapies. Annu Rev Pharmacol. 2019;59:191-211.

3. Bootzin RR, Caspi O. Explanatory mechanisms for placebo effects: cognition, personality and social learning. In: The science of the placebo; toward an interdisciplinary research agenda. London: BMJ Books, 2002:108-32.

4. Bardo MT. Neuropharmacological mechanisms of drug reward: Beyond dopamine in the nucleus accumbens. Crit Rev Neurobiol 1998;12:37-67.

5. Zunhammer M, Bingel U, Wager TD. Placebo effects on the neurologic pain signature: a meta-analysis of individual participant functional magnetic resonance imaging data. JAMA neurology. 2018;75(11):1321-30.

6. Hall KT, Loscalzo J, Kaptchuk TJ. Genetics and the placebo effect: the placebome. Trends Mol Med. 2015;21(5):285-94.

7. Colagiuri B, Schenk LA, Kessler MD, Dorsey SG, Colloca L. The placebo effect: from concepts to genes. Neuroscience. 2015;307:171-90.

8. Holmes RD, Tiwari AK, Kennedy JL. Mechanisms of the placebo effect in pain and psychiatric disorders. Pharmacogenomics J. 2016; 16: 491-500.

9. Büchel C, Geuter S, Sprenger C, Eippert F. Placebo analgesia: a predictive coding perspective. Neuron. 2014;81(6):1223-39.

10. Kaptchuk TJ, Kelley JM, Conboy LA, et al. Components of placebo effect: randomised controlled trial in patients with irritable bowel syndrome. BMJ. 2008;336(7651):999-1003.

11. Enck P, Bingel U, Schedlowski M, Rief W. The placebo response in medicine: minimize, maximize or personalize?. Nat Rev Drug Discov. 2013;12(3):191-204.

12. Kradin R. The placebo response complex. J Anal Psychol 2004;49:617-634.

13. Buckalew LW, Coffield KE. An investigation of drug expectancy as a function of capsule color and size and preparation form. J Clin Psychopharmacol. 1982;2:245-248.

14. Sallis RE, Buckalew LW. Relation of capsule color and perceived potency. Percept Mot Skills 1984;58:897-8.

15. de Craen AJ, Tijssen JG, de Gans J, Kleijnen J. Placebo effect in the acute treatment of migraine: Subcutaneous placebos are better than oral placebos. J Neurol 2000;247:183-188.

16. Wall PD. Pain and the placebo response. In: Experimental and Theoretical Studies of Consciousness. New York:Wiley, 1993:187-216.

17. Colloca L, Benedetti F. Placebos and painkillers: is mind as real as matter?. Nat Rev Neurosci. 2005;6(7):545-52.

18. Blasini M, Peiris N, Wright T, Colloca L. The role of patient-practitioner relationships in placebo and nocebo phenomena. In: International review of neurobiology. Massachusetts: Academic Press, 2018;139:211-231.

19. Benedetti F, Durando J, Vighetti S. Nocebo and placebo modulation of hypobaric hypoxia headache involves the cyclooxygenase-prostaglandins pathway. PAIN@. 2014;155(5):9218.

20. Darnall BD, Colloca L. Optimizing placebo and minimizing nocebo to reduce pain, catastrophizing, and opioid use: a review of the science and an evidence-informed clinical 
toolkit. In: International review of neurobiology. Massachusetts: Academic Press, 2018;139:129-157.

21. Dworkin RH, Turk DC, Peirce-Sandner S, et al. Research design considerations for confirmatory chronic pain clinical trials: IMMPACT recommendations. PAIN@. 2010;149(2):177-93.

22. Greene CS, Goddard G, Macaluso GM, Mauro G. Topical review: placebo responses and therapeutic responses. How are they related?. J Orofac Pain. 2009;23(2).

23. Horing B, Weimer K, Muth ER, Enck P. Prediction of placebo responses: a systematic review of the literature. Front Psychol. 2014;5:1079.

24. Harris RE, Williams DA, McLean SA, et al. Characterization and consequences of pain variability in individuals with fibromyalgia. Arthritis Rheum. 2005;52(11):3670-4.

25. Farrar JT, Troxel AB, Haynes K, et al. Effect of variability in the 7-day baseline pain diary on the assay sensitivity of neuropathic pain randomized clinical trials: an ACTTION study. Pain. 2014;155(8):1622-31.

26. Smith SM, Amtmann D, Askew RL, et al. Pain intensity rating training: results from an exploratory study of the ACTTION PROTECCT system. Pain. 2016;157(5):1056-64.

27. Gewandter JS, Dworkin RH, Turk DC, et al. Improving study conduct and data quality in clinical trials of chronic pain treatments: IMMPACT recommendations. J Pain. 2019.

28. Zilcha-Mano S, Barber JP. Instability of depression severity at intake as a moderator of outcome in treatment for major depressive disorder. Psychother Psychosom. 2014;83(6):382.

29. Treister R, Eaton TA, Trudeau JJ, Elder H, Katz NP. Development and preliminary validation of the focused analgesia selection test to identify accurate pain reporters. $J$ Pain Res 2017;10:319-26.

30. Treister R, Lawal OD, Shecter JD, et al. Accurate pain reporting training diminishes the placebo response: results from a randomised, double-blind, crossover trial. PLoS One 2018;13:e0197844.

31. Treister R, Suzan E, Lawal OD. Staircase-evoked pain may be more sensitive than traditional pain assessments in discriminating analgesic effects: a randomized, placebocontrolled trial of naproxen in patients with osteoarthritis of the knee. Clin J Pain 2019;35:50-5.

32. Barrett LF, Simmons WK. Interoceptive predictions in the brain. Nat Rev Neurosci 2015;16:419-29.

33. Seth AK, Friston KJ. Active interoceptive inference and the emotional brain. Philos Trans R Soc B Biol Sci 2016;371:20160007.

34. Hoskin R, Berzuini C, Acosta-Kane D, El-Deredy W, Guo H, Talmi D. Sensitivity to pain expectations: a Bayesian model of individual differences. Cognition 2019;182:127-39.

35. Clark A. Whatever next? Predictive brains, situated agents, and the future of cognitive science. Behavioral and brain sciences. 2013;36(3):181-204.

36. Friston K. Learning and inference in the brain. Neural Networks. 2003;16(9):1325-52.

37. Brown CA, Seymour B, Boyle Y, El-Deredy W, Jones AK. Modulation of pain ratings by expectation and uncertainty: Behavioral characteristics and anticipatory neural correlates. Pain. 2008;135(3):240-50.

38. Colloca L, Petrovic P, Wager TD, Ingvar M, Benedetti F. How the number of learning trials affects placebo and nocebo responses. Pain ${ }^{\circledR}$. 2010;151(2):430-9. 
39. Lui F, Colloca L, Duzzi D, Anchisi D, Benedetti F, Porro CA. Neural bases of conditioned placebo analgesia. PAIN®. 2010;151(3):816-24.

40. Anchisi D, Zanon M. A Bayesian perspective on sensory and cognitive integration in pain perception and placebo analgesia. PloS one. 2015;10(2).

41. Jung WM, Lee YS, Wallraven C, Chae Y. Bayesian prediction of placebo analgesia in an instrumental learning model. PloS one. 2017;12(2).

42. Treister R, Kliger M, Zuckerman G, Aryeh IG, Eisenberg E. Differentiating between heat pain intensities: the combined effect of multiple autonomic parameters. PAIN@. 2012;153(9):1807-14.

43. Hu L, Iannetti GD. Neural indicators of perceptual variability of pain across species. PNAS. 2019;116(5):1782-91.

44. Shields SA, Mallory ME, Simon A. The body awareness questionnaire: reliability and validity. J Pers Assess. 1989;53(4):802-15.

45. Scheier MF, Carver CS. The Self-Consciousness Scale: A Revised Version for Use with General Populations 1. J Appl Soc Psychol. 1985;15(8):687-99.

46. Altmann T, Roth M. The Self-esteem Stability Scale (SESS) for cross-sectional direct assessment of self-esteem stability. Front Psychol. 2018;9:91.

47. Scheier MF, Carver CS, Bridges MW. Distinguishing optimism from neuroticism (and trait anxiety, self-mastery, and self-esteem): a reevaluation of the Life Orientation Test. J Pers Soc Psychol. 1994;67(6):1063.

48. Pollo A, Benedetti F. The placebo response: neurobiological and clinical issues of neurological relevance. Prog Brain Res. 2009;175:283-94.

49. Roy M, Shohamy D, Daw N, Jepma M, Wimmer GE, Wager TD. Representation of aversive prediction errors in the human periaqueductal gray. Nat Neurosci. 2014;17(11):1607.

50. Grahl A, Onat S, Büchel C. The periaqueductal gray and Bayesian integration in placebo analgesia. Elife. 2018;7:e32930.

51. Tetreault P, Mansour A, Vachon-Presseau E, Schnitzer TJ, Apkarian AV, Baliki MN. Brain connectivity predicts placebo response across chronic pain clinical trials. PLoS Biol. 2016;14(10).

52. Vachon-Presseau E, Berger SE, Abdullah TB, Huang L, Cecchi GA, Griffith JW, Schnitzer TJ, Apkarian AV. Brain and psychological determinants of placebo pill response in chronic pain patients. Nat Commun. 2018;9(1):1-5.

53. Ashar YK, Chang LJ, Wager TD. Brain mechanisms of the placebo effect: an affective appraisal account. Annual review of clinical psychology. 2017;13:73-98.

54. Hashmi JA, Baria AT, Baliki MN, Huang L, Schnitzer TJ, Apkarian AV. Brain networks predicting placebo analgesia in a clinical trial for chronic back pain. PAIN®. 2012;153(12):2393-402.

55. Mouraux A, Iannetti GD. The search for pain biomarkers in the human brain. Brain. 2018;141(12):3290-307.

56. Tétreault P. Can Functional Brain Connectivity Predict Placebo Response in Chronic Pain?. Clin Pharmacol Ther. 2019;106(6):1171-4. 
Figure 1
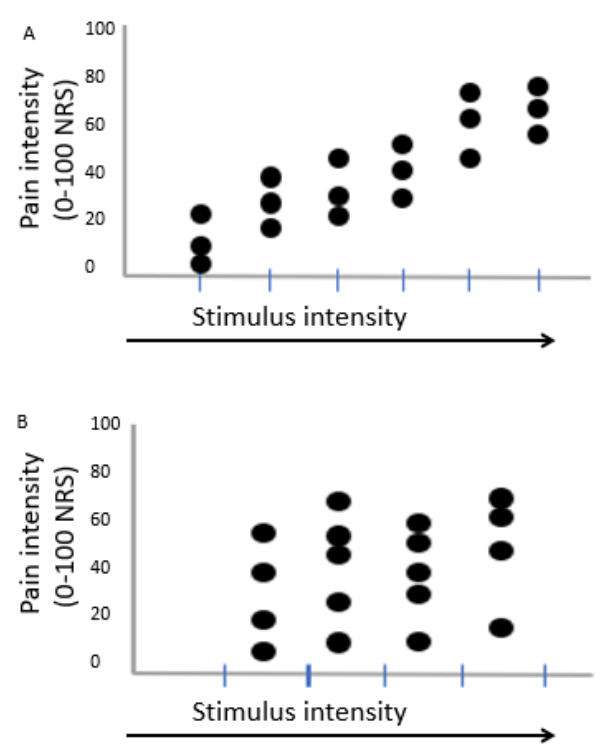
Figure 2

Experimental Pain Reports Clinical Pain Reports Placebo Response (FAST)
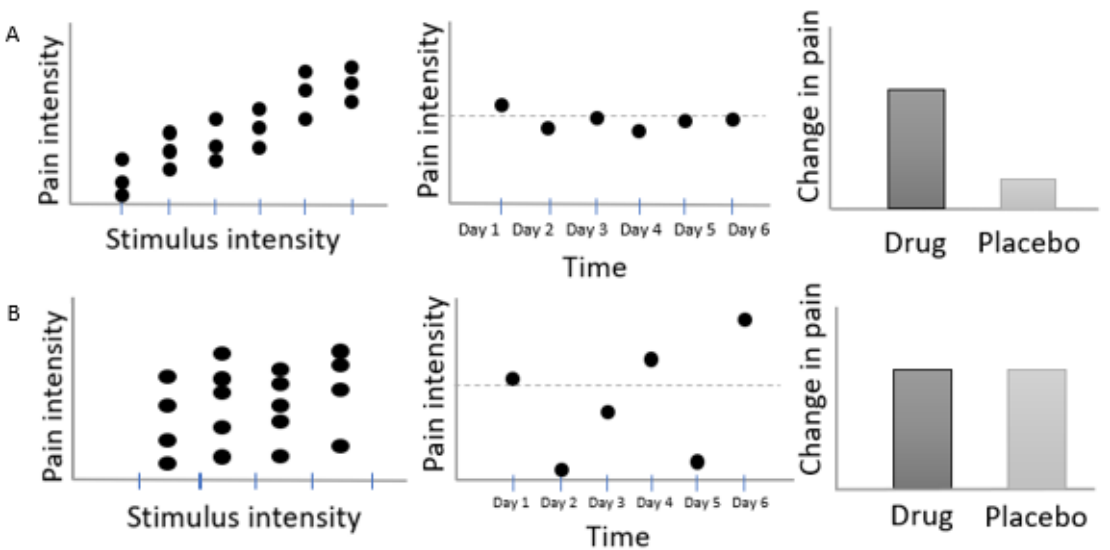
Figure 3

A

equal precision prior and likelihood

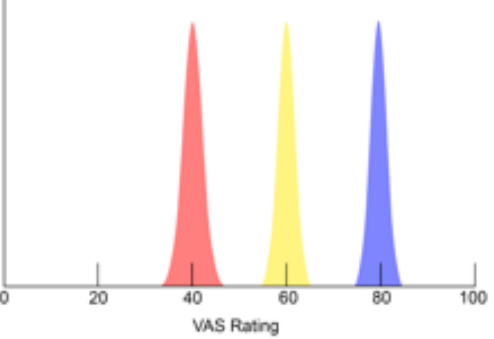

B

high precision prior, low precision likelihood

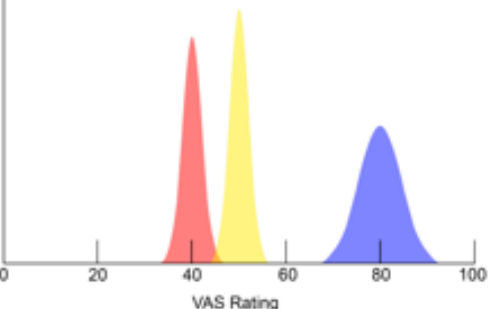

low precision prior, high precision likelihood

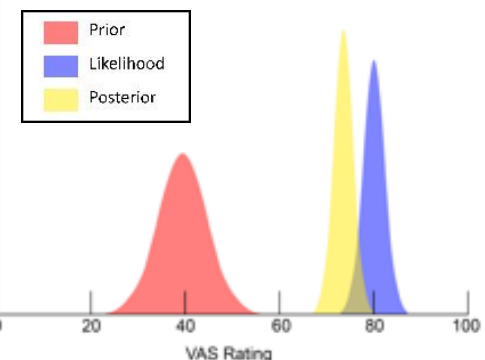


Figure 4
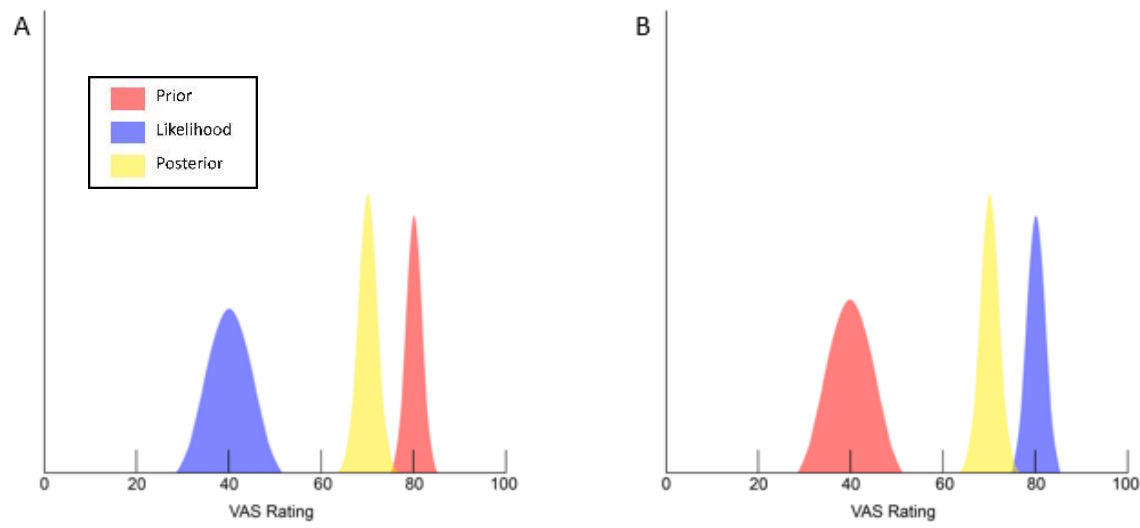


\section{FIGURE LEGENDS}

\section{Fig 1.}

A Illustrates the results from a patient who presents small variability in the FAST

B Illustrates the results from a patient who presents large variability in the FAST

Fig 2.

A Illustrates the results from a patient who presents small variability in the FAST, demonstrates reduced clinical pain variability, and has a low placebo response

B Illustrates the results from a patient who presents large variability in the FAST, demonstrates increased clinical pain variability, and has a high placebo response

\section{Fig 3.}

In these 3 figures, the $\mathrm{X}$ axis represent pain scores and the $\mathrm{Y}$ axis represents probability. Each of the 3 curves represent a component of the $\mathrm{BBH}$ (prior, posterior, likelihood). The priors represent expectations, the likelihood represent the sensory input, and the posteriors represent the perception. The intensity of each function is below the peak of each curve, and the precision of each function is inversely represented by the variance of each curve (large variance $=$ low precision, and vice versa). In all 3 figures, the intensities of the priors (red) and of the likelihood (purple) remain constant. The difference between the figures is the confidence of the priors and the likelihood. The curve of the posterior is compiled from the other two curves.

A Illustrates an example of a patient who has an equal confidence in their previous beliefs (prior 40/100) and in the incoming sensory information (likelihood 80/100) therefore perceiving the stimulus (posterior) at an intensity between the two (60/100).

B Illustrates an example of a patient who has a high confidence in their previous beliefs (prior 40/100), but low confidence in the incoming sensory information (likelihood 80/100) therefore perceiving the stimulus (posterior) at an intensity closer to that of the prior (50/100).

C Illustrates an example of a patient who has a low confidence in their previous beliefs (prior 40/100), and high confidence in the incoming sensory information (likelihood 80/100) therefore perceiving the stimulus (posterior) at an intensity closer to that of the likelihood (70/100).

\section{Fig 4.}

A and B represent two patients, both suffering from pain intensity $70 / 100$, but with different underlying causes.

A Illustrates an example of a patient with a high confidence in their expectation (prior) for pain of 80/100, and a low confidence in the incoming sensory information (likelihood) of 40/100, therefore perceiving the pain at a level closer to that of their expectation (70/100). 
B Illustrates a contrasting patient who expects lower pain (40/100), but with low certainty of priors and an incoming sensory signal of $80 / 100$, therefore their experience is closer to that of the likelihood (70/100). 